\title{
Social Media and its Impact on the General and Colorectal Surgery Communities
}

\section{Sosyal Medyanın Genel ve Kolorektal Cerrahi Topluluğuna Etkisi}

\author{
(1) Ali Cihat Yıldırım1, ㅁ Mehmet Fatih Ekici2 \\ ${ }^{1}$ Kütahya Health Sciences University Faculty of Medicine, Department of General Surgery, Kütahya, Turkey \\ ${ }^{2}$ Kütahya Health Science University Medical Faculty Evliya Çelebi Hospital, Clinic of General Surgery, Kütahya, Turkey
}

Keywords: Colorectal surgery, social media, some4surgery

Anahtar Kelimeler: Kolorektal cerrahi, sosyal media, ameliyat

\section{Dear Editor,}

Since early in the year 2020, the Coronavirus disease-19 (COVID-19) pandemic has evolved and is affecting every aspect of life, including surgical healthcare worldwide. Surgical practice and surgery have been affected mostly due to their dependency on practical education. ${ }^{1}$ Social media, which is increasingly being used nowadays, has been supporting surgical education through free educational contents, video platforms, easy to communicate mentorship facilities and international collaborations without borders. Therefore, on the popular social media site twitter, surgical experts formed "SoMe4Surgery" and embedded twitter communities like "SoMe4Proctology". They use hashtags (\#) and tweet with hashtags like "\#SoMe4Surgery, \#SoMe4ColorectalSurgery", etc. ${ }^{2}$ One of this popular online educational platforms was created by young academic surgeons and handled by residents from El Bosque University, Bogota, Colombia. These young surgeons have as primary aim to support the free share of surgical learning by tagging well-known surgical experts to the daily surgical cases and educational materials that are sent by tweeting the related literature, studies and surgical illustrations. Great incorporation has been seen by responses to daily surgical cases when many surgeons have been reported their own comments, and a tremendous educational era has been seen inter-continentally. ${ }^{3}$

Since 2016, colorectal surgeons have been among the leaders in social media use, especially twitter, due to the creation of the hashtag "colorectal surgery", and a growing impact was created by 100,000 tweet impressions. Colorectal surgeons who had been attending and live tweeting colorectal surgery specific meetings had been sharing scientific contents with colorectal surgery hashtag; therefore, this has enabled many surgeons globally to learn the most up-to-date data from experts. ${ }^{4}$

One of the benefits of social media use by surgeons is to facilitate research collaborations, in which high-quality peerreviewed research protocols are developed through easy and fast communication among young medical students, residents and surgeons with minimal costs. One of these groups have developed the Global Surg network, which later has been published as one of the most essential COVID-19 surgical study to date; from "COVIDSurg" initiative on this background. $^{5}$

Another positive effect of the articles shared on social media was analysed by Korean surgeons and their study was found that article exposure on Twitter leads to a high citation impact. ${ }^{6}$

However, there are also growing concerns about social media use and sharing of information among surgeons, which can be summarised into four main categories as misrepresentation of non-peer-reviewed data, sharing of copyright-protected content, the spread of biased information and damage to professional image. ${ }^{4}$ Therefore, creators of surgical education platforms try to form restricted groups that only enable to 
access surgeons, residents and medical students. Another ethical debate about sharing patient's data by imaging and videos even without any special identification of that person is still ongoing.

Additionally, surgeons should be aware that not every surgical content is feasible or optimal to be accepted as a learning material. ${ }^{7}$ Therefore some surgical communities create guidelines for members who want to use social media for training purposes. According to these principles, it is strongly recommended that trainers and trainees of social media should comply with the local laws protecting patient privacy. It is also recommended that surgical associations educate and inform especially trainers in social media to weigh the costs and odds of it. Therefore, the society of gastrointestinal and endoscopic surgeons made taskforce recommendations for closed-content Facebook groups, stating that obtaining the patients' informed consent is still one of the physicians' responsibilities on social media. ${ }^{8}$

In conclusion, professional and ethical use of social media can help the surgical community in all aspects like surgical education, collaboration and dissemination lasted up-todate scientific evidence-based materials. Considering all of its benefits, surgeons should also be aware of the potential risks and should act and behave according to local and universal regulations as mentioned above.

Peer-review: Internally peer reviewed.

\section{Authorship Contributions}

Surgical and Medical Practices: A.C.Y., Concept: A.C.Y., Design: A.C.Y., Data Collection or Processing: M.F.E,
Analysis or Interpretation: M.F.E., Literature Search: M.F.E., Writing: A.C.Y., M.F.E

Conflict of Interest: No conflict of interest was declared by the authors.

Financial Disclosure: The authors declared that this study received no financial support.

\section{References}

1. Wexner SD, Cortés-Guiral D, Gilshtein H, Kent I, Reymond MA. COVID-19: impact on colorectal surgery. Colorectal Dis. 2020;22:635-640.

2. Ioannidis A, Blanco-Colino R, Chand M, et al. How to make an impact in surgical research: a consensus summary from the \#SoMe4Surgery community [published online ahead of print, 2020 May 2]. Updates Surg. 2020;10.1007/s13304-020-00780-z. doi:10.1007/s13304-020-00780zCirbosque yayin

3. Mayol J, Otero J. Breaking International Barriers: \#ColorectalSurgery Is \#GlobalSurgery. Clin Colon Rectal Surg. 2017;30:277-280.

4. COVIDSurg Collaborative. Mortality and pulmonary complications in patients undergoing surgery with perioperative SARS-CoV-2 infection: an international cohort study [published correction appears in Lancet. 2020;396:27-38.

5. Wexner SD, Petrucci AM, Brady RR, Ennis-O'Connor M, Fitzgerald JE, Mayol J. Social media in colorectal surgery. Colorectal Dis. 2017;19:105114.

6. Jeong JW, Kim MJ, Oh HK, et al. The impact of social media on citation rates in coloproctology. Color Dis. 2019;21:1175-1182.

7. Lima DL, Cordeiro RN, Carvalho GL, Malcher F. The influence of social media in minimally invasive surgery education: How surgeons exchange experience and knowledge in these platforms. J Minim Access Surg. 2019;15:275-276

8. Bittner JG 4th, Logghe HJ, Kane ED, et al. A Society of Gastrointestinal and Endoscopic Surgeons (SAGES) statement on closed social media (Facebook®) groups for clinical education and consultation: issues of informed consent, patient privacy, and surgeon protection. Surg Endosc. 2019;33:1-7. 